\title{
A Packet Scheduling Scheme for Audio-Video Transmission over Error-Prone IEEE 802.11e HCCA Wireless LANs
}

\author{
Zul Azri Bin Muhamad Noh ${ }^{\dagger} \quad$ Takahiro Suzuki ${ }^{\dagger \dagger}$ Shuji Tasaka ${ }^{\dagger}$ \\ ${ }^{\dagger}$ Graduate School of Engineering, Nagoya Institute of Technology, Japan \\ ${ }^{\dagger \dagger}$ Faculty of Health Sciences, Nihon Fukushi University, Japan \\ Email: ${ }^{\dagger}\left\{\right.$ zulazri,tasaka\}@inl.nitech.ac.jp ${ }^{\dagger \dagger}$ suzuki@n-fukushi.ac.jp
}

\begin{abstract}
This paper proposes a packet scheduling scheme for QoS support in audio-video transmission with IEEE 802.11e HCCA. In the proposed scheme, the access point (AP) basically allocates transmission opportunity (TXOP) for each station like the reference scheduler of the IEEE 802.11e standard, which is referred to as the TGe scheme in this paper. However, the AP with the proposed scheme can allocate additional TXOP in a service interval $(S I)$ if the $\mathbf{A P}$ has received corrupted $M A C$ protocol data units (MPDUs) in the previous SI. In addition, the proposed scheme calculates the number of $M A C$ service data units $(M S D U s)$ arrived in an SI, considering the inter-arrival time of audio samples and that of video frames, which are referred to as media units $(M U s)$, at the application-layer. We then compare the proposed scheme with the TGe scheme in terms of applicationlevel $\mathrm{OoS}$ in the case where stations transmit audio and video flows to the AP in a noisy environment. Numerical results show that the proposed scheme improves the average MU delay and media synchronization quality even if channel transmission error occurs to some extent. Since the proposed scheme uses the surplus bandwidth for MPDU retransmission, we also discuss the number of admitted stations in the scheme.
\end{abstract}

\section{INTRODUCTION}

Provisioning quality of service $(Q o S)$ guarantee for multimedia transmission by IEEE 802.11 wireless LAN (WLAN) is achieved through introduction of the IEEE 802.11e hybrid coordination function $(H C F)$. The HCF consists of two channel access methods: enhanced distributed channel acces (EDCA) and HCF controlled channel access (HCCA) [1]. The former extends contention-based distributed coordination function $(D C F)$ by providing priority-based service differentiation. The latter enhances polling-based point coordination function $(P C F)$ by supporting guaranteed medium access for traffic flows based on their QoS requirements. The random access of EDCA provides only service differentiation between the traffic types within an basic service area (BSA) and cannot provide strict bandwidth guarantee. Therefore, in delay constraint multimedia transmission, centrally controlled HCCA is preferred.

In the HCCA, QoS requirements must be met once a traffic flow has been admitted through its admission control; thus, HCCA gives high assurance of QoS guarantee. In this paper, we focus on the audio-video transmission with the HCCA.

In the HCCA, QoS support is achieved through packet scheduling and admission control. For reference, the IEEE $802.11 \mathrm{e}$ standard has presented an example packet scheduler, which is referred to as the Task Group e (TGe) scheme in this paper.

In the TGe scheme, the transmission opportunity (TXOP) duration for a station is calculated by the access point (AP) on the basis of the traffic specification (TSPEC) information received from the station. The TSPEC consists the set of parameters that define the characteristics and QoS expectations of a traffic flow like the mean data rate, delay bound, nominal $M A C$ service data unit (MSDU) size, and maximum service interval (MSI). For simplifying the description of the TGe scheme, let us focus on flow $i$ where $\rho_{i}$ is the mean data rate in bits per second, $L_{i}$ is the nominal MSDU size in bits, and $R_{i}$ is the physical transmission rate of flow $i$ in bits per second.

The AP first determines the service interval (SI), which is the interval between two successive service periods. In other words, the AP polls a station once every SI. The SI is a value lower than the smallest value among the MSI of admitted stations that is a submultiple of the beacon interval. If a new flow is admitted with an MSI smaller than the current SI, the AP needs to change the current SI to smaller value than the MSI of the newly admitted station.

Then, the AP calculates the number of MSDU of flow $i$ that arrives at the mean data rate during an SI as

$$
N_{i}^{T}=\left\lceil\frac{S I \times \rho_{i}}{L_{i}}\right\rceil
$$

where $S I$ is the duration of the SI. The superscript $T$ means the TGe scheme. Then, the TXOP duration for flow $i$ is computed as

$$
T X O P_{i}^{T}=\max \left(\frac{N_{i}^{T} \times L_{i}}{R_{i}}+O, \frac{M}{R_{i}}+O\right)
$$

where $M$ is the maximum allowable size of MSDU, and $O$ is the overhead in time units due to the physical header, MAC header, inter-frame space (IFS), acknowledgment frames, and poll frames.

In the TGe scheme, the AP calculates the TXOP duration for each station on the basis of the mean data rate and nominal MSDU size. This implies that a station is allocated fixed TXOP duration in every SI; therefore this scheme is suitable for transmission of constant bit rate $(C B R)$ traffic with error-free channel. However, the TGe scheme has three disadvantages, which are mentioned below.

First, the TGe scheme cannot guarantee QoS for variable bit rate $(V B R)$ traffic because it does not take the data rate and packet size fluctuation into account.

Secondly, in the TGe scheme, the TXOP duration for each station is calculated on the assumption that the channel is error-free. However, transmission errors can occur in WLANs owing to shadowing, multipath fading, and interference. In this case, the TXOP duration becomes insufficient because channel capacity for retransmission traffic is not allocated. In wireless multimedia transmission, packet scheduling should be designed so as to accommodate retransmission traffic as well as newly generated traffic to improve QoS in a noisy environment.

Thirdly, the derived number of arriving MSDUs calculated from Eq. (1) becomes smaller than the real number; this leads to insufficient channel allocation in the TGe scheme. For example, in the case of video flow used in our study, one video frame is generated in every $50 \mathrm{~ms}$, the mean date rate $\rho$ is 800 
kbps and nominal MSDU size $L$ is 1500 bytes; therefore, the video frame is fragmented into 4 MSDUs. Thus, 8 MSDUs are generated in an SI when SI is $100 \mathrm{~ms}$. On the other hand, according to Eq. (1), the number of arriving MSDUs becomes 7 MSDUs if SI is $100 \mathrm{~ms}$. Note that this problem occurs since the derived number of arriving MSDUs in an SI is calculated from only MAC-level parameters, though the real number of arriving MSDUs highly depends on video frame rate at the application-layer.

There are numerous studies trying to improve the inefficiency of the TGe scheme. In [2]-[7], various methods are proposed to improve QoS for transmission of VBR traffic. However, these studies have been focusing only on MAClevel QoS; that is, the QoS is assessed in terms of the MAClevel throughput and MAC frame delay. In addition, references [2] and [7] examine MAC-level QoS considering channel transmission error. However, these studies have not explicitly discussed surplus bandwidth allocation for retransmission traffic.

With regard to QoS at upper levels, reference [8] examines the application-level QoS of video transmission with a resource allocation scheme in terms of peak signal-to-noise ratio (PSNR). In the scheme, one video flow is sent as more than one MAC flow. In addition, reference [9] proposes the multimedia priority dynamic scheduling (MPDS) scheme for transmission of VBR traffic and have assessed applicationlevel QoS and quality of experience $(Q o E)$ in the case where audio and video are transferred from stations to the AP in a error-free channel.

In this paper, we try to solve the second and third problems of the TGe scheme described earlier to support QoS of audio and video transmission by the IEEE 802.11e HCCA. To cope with the second problem, we design a simple strategy to allocate surplus bandwidth for MAC protocol data unit $(M P D U)$ retransmission in the TGe scheme. In the proposed scheme, the AP allocates additional TXOP duration in an SI on the basis of the number of corrupted MPDUs in the previous SI. Furthermore, the proposed scheme calculates the number of MSDUs generated by a station in an SI, using the generation interval of audio samples and that of video frames at the application-layer to overcome the third problem.

We then evaluate the application-level QoS of the proposed scheme and the TGe scheme through simulation. Applicationlevel QoS is important in multimedia transmission since multimedia flows like audio and video have temporal structure to be preserved. In this paper, the media synchronization quality is regarded as the major part of the application-level QoS. We examine the effect of SI and maximum number of retransmission on the QoS and discuss the superiority of the proposed scheme to the TGe scheme. We also show the relationships between the number of stations and the application-level QoS to discuss an admission control scheme in a noisy environment.

The rest of the paper is organized as follows. Section II explains the mechanism of HCCA and admission control in the TGe scheme. In Section III, we present the proposed scheme. Next in Section IV, we specify the simulation conditions. Section V presents numerical results. Finally, Section VI concludes this paper.

\section{IEEE 802.11E HCCA MECHANISM}

In this section, we introduce the mechanism of HCCA and the method of admission control in the TGe scheme.

\section{A. IEEE 802.11e HCCA Channel Access}

In the IEEE 802.11e HCCA WLAN, the contention free period $(C F P)$ and contention period $(C P)$ alternate periodically over time, and a combination of CFP and CP forms a superframe, which starts with a beacon frame. The CFP and $\mathrm{CP}$ are used for the HCCA and EDCA, respectively.

A station which requires QoS guarantee must query for a QoS reservation by sending its TSPEC to the AP. The AP then determines whether the QoS reservation request made by the station can be admitted or should be rejected through its admission control unit (ACU). If the AP admits the QoS reservation request, it allocates TXOP to the station on the base of a scheduling scheme.

\section{B. Admission Control in the TGe Scheme}

Owing to the limitation of the capacity during an SI, the TGe scheduler implements admission control to ensure that all admitted flows have adequate TXOP duration for their QoS Admission control decides which flow should be admitted and which flow should be dropped from the polling list. When flow $k+1$ issues a QoS reservation, the AP will first check whether the available capacity of the medium exists or not by the following equation:

$$
\frac{T X O P_{k+1}^{T}}{S I}+\sum_{i=1}^{k} \frac{T X O P_{i}^{T}}{S I} \leq \frac{T-T_{C P}}{T}
$$

where $T$ is the beacon interval, and $T_{C P}$ is the time for the EDCA. If Eq. (3) is satisfied, the AP admits flow $k+1$ into its polling list and allocates TXOP to the flow.

\section{Proposed Scheduling Scheme}

In this paper, we propose a method of allocating additional TXOP duration. The proposed scheme is designed to fulfill insufficient TXOP duration in the event of transmission error.

In the proposed scheme, the AP first calculates the basic TXOP duration in a similar way to that of the TGe scheme but with replacement of Eq. (1). In order to derive the number of MSDUs generated in an SI accurately, we propose a new method for calculating the number of MSDUs.

In the proposed method, information about inter-MU (media unit $^{1}$ time is required. This is because the video frame is generated periodically and fragmented into several MSDUs. Therefore, the calculation based on an inter-MU time can give an accurate value of the number of MSDUs. Using the inter-MU time together with the TSPEC, we first compute the number of MSDUs arriving within an inter-MU time as

$$
n_{i}^{P}=\left\lceil\frac{i n t e r \_M U_{i} \times \rho_{i}}{L_{i}}\right\rceil
$$

where inter $M U_{i}$ denoted the inter-MU time for flow $i$. The superscript $\bar{P}$ means the proposed scheme. Then, the number of MSDUs that arrives in an SI is computed as

$$
N_{i}^{P}=\left\lceil\frac{S I}{\text { inter_MU } \_} \times n_{i}^{P}\right\rceil
$$

Then, the basic TXOP duration is calculated with Eq. (2) by replacing $N_{i}^{T}$ with $N_{i}^{P}$

On top of the basic TXOP duration, the AP then allocates additional TXOP duration. The AP keeps monitoring the number of corrupted MPDU owing to transmission errors in every SI. In the event of transmission error, the AP does not acknowledge the corrupted MPDU, and the retransmission counter of the corresponding station will be incremented. If the AP has received $E$ (say) corrupted MPDUs from a station in an SI, the AP allocates additional TXOP duration to the station for retransmission of $E$ MPDUs with the nominal MSDU size in the next SI. If the AP receives corrupted MPDUs, it cannot distinguish the flow types between audio and video. For this reason, the AP allocates additional TXOP duration for transmission of MPDUs with the nominal MSDU size of the video flow, which has a bigger MSDU size than that of audio.

As the surplus bandwidth reduces with the increase of admitted flows, the AP might not be able to allocate enough additional TXOP duration. In this case, the AP distributes the surplus bandwidth for stations in a round-robin basis; that is,

\footnotetext{
${ }^{1}$ The transmission unit of data at the application layer. A video MU is
} defined as a video frame and is transferred as one or more UDP datagrams. 
additional TXOP duration for retransmission of one nominalsized MSDU will be allocated to each station until the surplus bandwidth is fully occupied. Note that the maximum additional TXOP duration for a station in an SI is the value of $E$ for the station.

\section{Simulation CONDitions}

Figure 1 illustrates the system configuration used in the simulation. We focus on a single basic service set (BSS) which includes an AP and several multimedia stations. We assume the IEEE 802.11b physical layer based on direct sequence spread spectrum (DSSS) with a channel data rate of $11 \mathrm{Mb} / \mathrm{s}$ [10]. In the simulation, two types of traffic are considered: audio and video. Each multimedia station sends stored audio and video streams to the AP as two separate transport streams using UDP/IP.

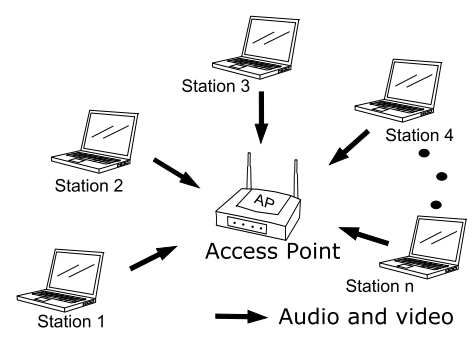

Fig. 1. System Configuration

Table 1 summarizes media specifications of audio-video flows used in the simulation. We use an audio flow of ITU-T G.711 $\mu$-law and an H.264 video flow. An $M U$ stands for a "media unit", which indicates the information unit for media synchronization [11] at the application layer. A video MU is defined as a video frame and is transferred as one or more UDP datagrams. An audio MU consists of 1000 audio samples, which corresponds to a single UDP datagram. The audio bit rate is constant at $64 \mathrm{~kb} / \mathrm{s}$, and the video bit rate is $800 \mathrm{~kb} / \mathrm{s}$ on average.

We use Music video as the video content. The Music video shows scenes of a Japanese female singer dancing while singing.

TABLE I

SPECIFICATIONS OF AUdIO AND VIDEO

\begin{tabular}{l|c|c}
\hline & Audio & Video \\
\hline \hline coding scheme & G.711 $\mu$-law & H.264 \\
\hline image size [pixel] & - & $320 \times 240$ \\
\hline picture pattern & - & $\mathrm{I}$ \\
\hline average MU size [byte] & 1000 & 5000 \\
\hline average MU rate [MU/s] & 8 & 20 \\
\hline average inter-MU time [ms] & 125 & 50 \\
\hline average bit rate [kb/s] & 64 & 800 \\
\hline measurement time [s] & 60 & 60 \\
\hline
\end{tabular}

In the simulation, we set the beacon interval to $1000 \mathrm{~ms}$. The nominal MSDU size for audio and that for video are set to 1000 bytes and 1500 bytes, respectively. Moreover, the controlled access period $(C A P)$ ratio is 0.8 , which means at most $80 \%$ of the bandwidth within SI is allocated for the HCCA.

In modeling of the wireless transmission error, we utilize the signal-to-noise ratio (SNR) based on Orinoco $802.11 \mathrm{~b}$ Card [12] and use an empirical curve of bit error rate (BER) versus SNR provided by Intersil WLAN chipset [13]. In the simulation, we assume that the distance between the AP and each multimedia station is the same and is set to $150 \mathrm{~m}$, $160 \mathrm{~m}$, and $170 \mathrm{~m}$, which correspond to BER of $2.4 \times 10^{-5}$, $4.1 \times 10^{-5}$, and $7.0 \times 10^{-5}$, respectively.

\section{NumERICAL RESULTS}

In this section, we compare the TGe scheme and the proposed scheme in terms of application-level QoS in audiovideo transmission. We first study the effect of SI on the application-level QoS. Next, we assess the effect of maximum number of retransmission on the QoS. Then, we evaluate how the number of admitted stations affects the QoS.

In the case of the TGe scheme, the calculation of arriving number of MSDUs can be inaccurate as mentioned in Sec. I. Therefore, we also introduce a modified TGe scheme where the arriving number of MSDUs in an SI is calculated by Eq. (5), in the same way as the proposed scheme. In the modified TGe scheme, however, additional TXOP duration for retransmission traffic is not allocated. In the following numerical results, TGe' means the modified TGe scheme.

\section{A. The Effect of SI}

First, we study the effect of SI on the application-level QoS. In the simulation, we set various values of MSI in order to derive the SI values ranging from $25 \mathrm{~ms}$ to $500 \mathrm{~ms}$. The number of multimedia stations is set to 4 . In addition, the maximum retry number for retransmission attempt is set to 4 . An MPDU will be discarded if this number is exceeded. In the figures to be shown, notation "TGe'[EF]", for instance, refers to the result of the modified TGe scheme on the error free $(E F)$ channel condition. Meanwhile, notation "Proposed[160]" refers to the results of the proposed scheme under a channel condition that the distance between the AP and the station is $160 \mathrm{~m}$.

Figure 2 shows the average MU delay for video as a function of the SI. The average MU delay is the average time from the moment an MU is generated at the source station until the moment the MU is output at the receiver. We can observe in this figure that the average MU delay for the TGe' scheme and that of the proposed scheme become very small for all the values of SI if the channel is error-free. Meanwhile, the average MU delay for the TGe scheme becomes larger and fluctuates if SI increases beyond $50 \mathrm{~ms}$. This is because the $\mathrm{TGe}$ scheme provides inaccurate numbers of arriving MSDUs from Eq. (1).

Table 2 shows the derived number of arriving MSDUs from Eq. (1), that from Eq. (5), and actual number of arriving MSDUs for an SI. In Table 2, we find that the proposed scheme derives the same number of arriving MSDUs as the actual number. However, the number in the TGe scheme becomes smaller than the actual one. This leads to an insufficient TXOP duration to transmit all MSDUs. As a result, the remainder of the MSDUs will accumulate in the source buffer; this increases the average MU delay.

Figure 2 also shows that the average MU delay for the TGe' scheme increases with the increase of BER. On the other hand, in the proposed scheme, the average MU delay does not become larger when SI is larger than $100 \mathrm{~ms}$ except the case of $170 \mathrm{~m}$. This is because in the proposed scheme additional TXOP duration is allocated. However, the average MU delay for $170 \mathrm{~m}$ becomes larger because many MPDUs are retransmitted. In addition, this figure reveals that the average MU delay in the proposed scheme increases if SI becomes smaller than $100 \mathrm{~ms}$ since the fraction of the surplus bandwidth to the SI is too small to allocate additional TXOP duration to all stations.

Figure 3 shows the coefficient of variation of output interval for video, which is defined as the ratio of the standard deviation of the MU output interval of the video flow to its average. This QoS parameter represents the smoothness of

TABLE II

NUMBER OF MSDUS

\begin{tabular}{l|l|l|l|l|l|l|l|l|l|l|l}
\hline SI (ms) & 25 & 50 & 71.4 & 100 & 125 & 143 & 167 & 200 & 250 & 333 & 500 \\
\hline \hline Eq. (1) & 2 & 4 & 5 & 7 & 9 & 10 & 12 & 14 & 17 & 23 & 34 \\
Eq. (5) & 2 & 4 & 6 & 8 & 10 & 12 & 14 & 16 & 20 & 27 & 40 \\
Actual & 2 & 4 & 6 & 8 & 10 & 12 & 14 & 16 & 20 & 27 & 40 \\
\hline
\end{tabular}




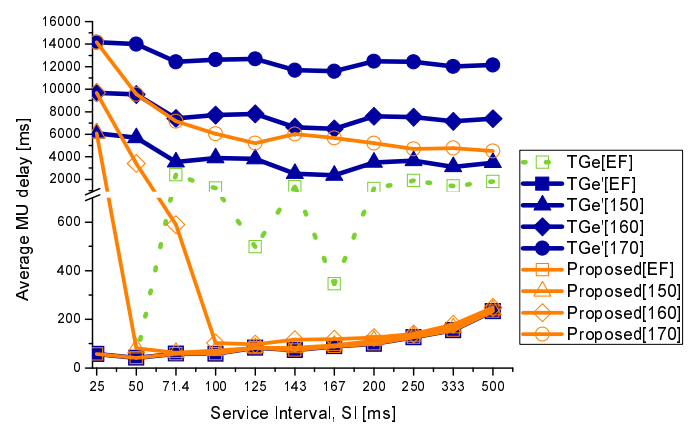

Fig. 2. Average MU delay for video

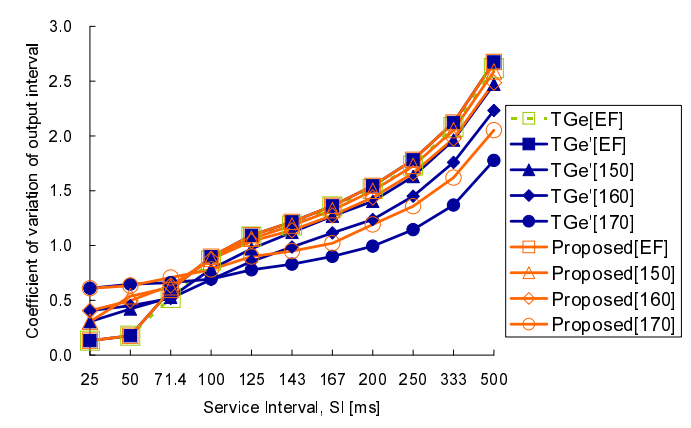

Fig. 3. Coefficient of variation of output interval for video

the output flow. This figure shows that for all schemes, the coefficient of variation of output interval increases with the increase of SI. As a video MU is generated in every $50 \mathrm{~ms}$, it is ideal to send the MU immediately after the generation. However, when the SI is above $50 \mathrm{~ms}$, several video MUs with different generation time are accumulated first before being sent. Therefore, the increase of SI will increase the number of accumulated MUs, which leads to increase of the coefficient of variation of output interval.

We have performed subjective experiment where we actually output the audio and video flows according to the output timing obtained from the simulation. From the subjective experiment, we have found that the subjective quality (QoE) for TGe' $[\mathrm{EF}]$ and that for Proposed[EF] are both good when the SI is below $100 \mathrm{~ms}$ over an error-free channel. However, the QoE for both schemes begins to deteriorate when the SI increases beyond $100 \mathrm{~ms}$ since the coefficient of variation of output interval becomes larger. We have also confirmed that the QoE for the TGe' scheme becomes lower than that for the proposed scheme in a noisy environment.

\section{B. The Effect of the Maximum Number of Retransmission}

Next, we examine the effect of the maximum number of retransmissions on the application-level QoS. In the simulation, we consider only one multimedia station, and the SI is set to $100 \mathrm{~ms}$, which is the minimum value where the applicationlevel QoS does not deteriorate owing to insufficient bandwidth to allocate additional TXOP duration. The maximum retry number for retransmission attempt is set from 0 to 5 . We compare the TGe' scheme and the proposed scheme.

In the figures to be shown, notation "TGe'-4" and "Proposed-4", for instance, refer to the results of the modified TGe scheme and the proposed scheme, respectively, when the maximum number of retransmission is set to 4 .

Figure 4 shows the MU loss ratio for video as a function of the distance between AP and each station. The MU loss ratio is the ratio of the number of MUs not output at the receiver to the number of MUs generated by the source station. In this figure, the MU loss ratio for the both scheme is approximately the same because the BER is the same. The MU loss in the simulation is due to the MPDU retransmission that exceeds the

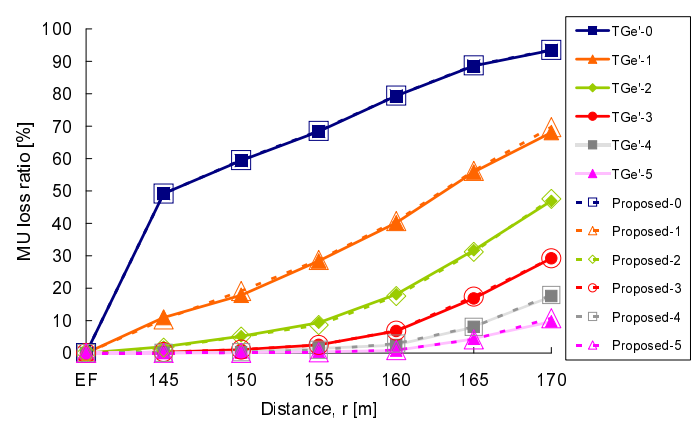

Fig. 4. MU loss ratio for video

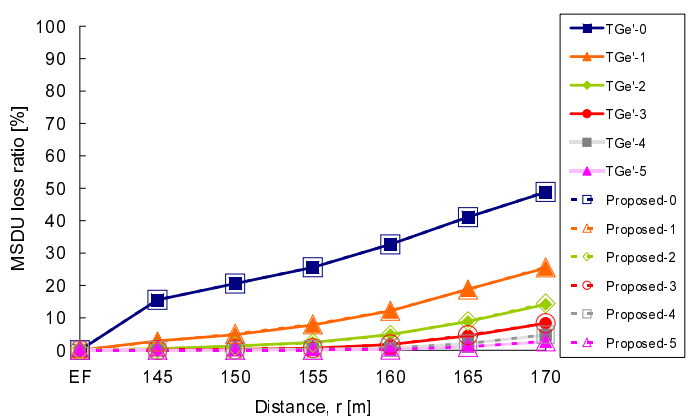

Fig. 5. MSDU loss ratio for video

maximum number of retransmission. We find in Fig. 4 that a larger maximum number of retransmission reduces the MU loss ratio. In addition, we have confirmed through simulation that the average MU delay for the proposed scheme is kept low even if the maximum number of retransmission increases, though the average MU delay for the TGe scheme deteriorates as the maximum number increases.

Meanwhile, Fig. 5 illustrates the MSDU loss ratio for video. This figure shows the same tendency to the MU loss ratio. However, we observe that the MU loss ratio takes higher values than the MSDU loss ratio. This is because a video MU is fragmented into several MSDUs. Therefore, the loss of one or more MSDUs of the fragmented MU leads to the loss of the corresponding whole MU.

In addition to the video with picture pattern $I$, we have also conducted a similar simulation with an IPPPPP picture pattern video. From the simulation results, we have observed that the MU loss ratio for the IPPPPP video becomes larger than those for the $I$ video. This is because a video frame is decoded after all frames within a group of picture $(G O P)$ are received correctly.

\section{The Effect of the Number of Admitted Stations}

Finally, we investigate the effect of the number of admitted stations on the application-level QoS. In this simulation, we set the SI to $100 \mathrm{~ms}$. Meanwhile, the maximum retry number for retransmission attempt is set to 4 . We increase the number of multimedia stations from 1 to 5 . It should be noted that on the simulation conditions the maximum number of admitted stations in the TGe' scheme is 5, which can obtain from Eq. (3).

Figures 6 and 7 indicate the average MU delay for audio and video, respectively, as a function of the number of multimedia stations. From these figures, we can observe that in the case of the TGe' scheme, the average MU delay for audio and that for video deteriorates as the distance increases. This is because additional TXOP duration is not allocated in the TGe' scheme.

From Fig. 6, we can find that the average audio MU delay for the proposed scheme becomes very small except for the case where the number of stations is five and the distance is $170 \mathrm{~m}$. Meanwhile, we can see in Fig. 7 that the average video MU delay for the proposed scheme begins to increase if the 


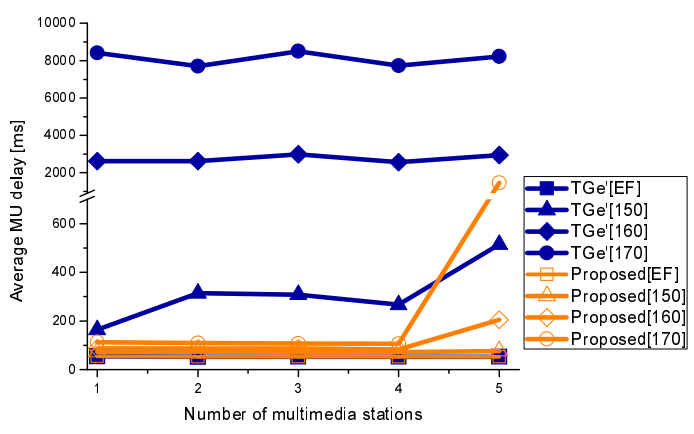

Fig. 6. Average MU delay for audio

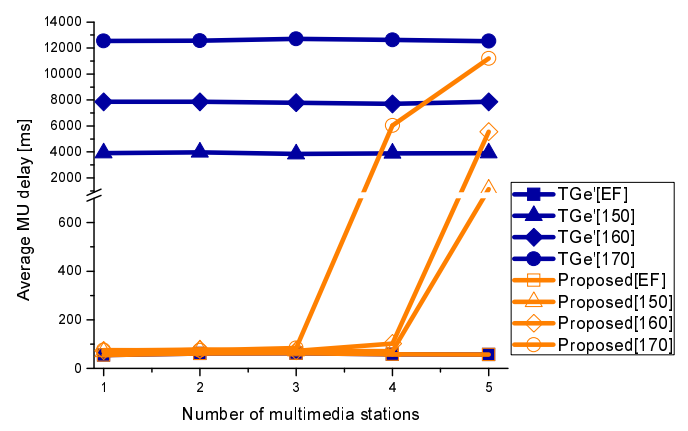

Fig. 7. Average MU delay for video

number of stations becomes larger than four for the distances $150 \mathrm{~m}$ and $160 \mathrm{~m}$, and three for $170 \mathrm{~m}$.

We also make a similar observation in Fig. 8, which plots the mean square error of inter-stream synchronization; it deteriorates even if the number of stations is less than or equal to 5 . The mean square error of inter-stream synchronization is an indicator of "lip-sync" and is the average square of the difference between the output time difference of the audio and the corresponding video MUs and their timestamp difference.

From the above observations, we can say that applicationlevel QoS for the proposed scheme deteriorates even if the number of stations is less than the maximum number of admitted stations calculated from Eq. (3). Therefore, a new admission control method is needed to provide QoS support in a lossy channel condition.

One simple method is that the AP monitors channel capacity for retransmission traffic for several SI's and estimates additional capacity for retransmission traffic. In this case, the admission control can be done as follows:

$$
\frac{T X O P_{k+1}^{P}+C_{k+1}^{P}}{S I}+\sum_{i=1}^{k} \frac{T X O P_{i}^{P}+C_{i}^{P}}{S I} \leq \frac{T-T_{C P}}{T}
$$

where $C_{i}^{P}$ is the estimated additional TXOP duration of flow $i$ for the current channel condition. We have confirmed through simulation that the maximum number of admitted stations for the distances $150 \mathrm{~m}, 160 \mathrm{~m}$, and $170 \mathrm{~m}$ are 4, 3, and 2, respectively.

\section{CONCLUSIONS}

We proposed a packet scheduling scheme for audio-video transmission by IEEE 802.11e HCCA and compared the application-level QoS of the TGe scheme, the TGe' scheme, and the proposed scheme. We studied the effect of SI, maximum number of retransmission, and number of multimedia stations on the application-level QoS. We have shown that in the TGe scheme the number of arriving MSDUs in an SI can be smaller than that of the actual ones. We have confirmed through simulation that the proposed scheme can improve the QoS of the TGe' scheme in an error-prone channel because the AP can utilize surplus bandwidth to allocate additional

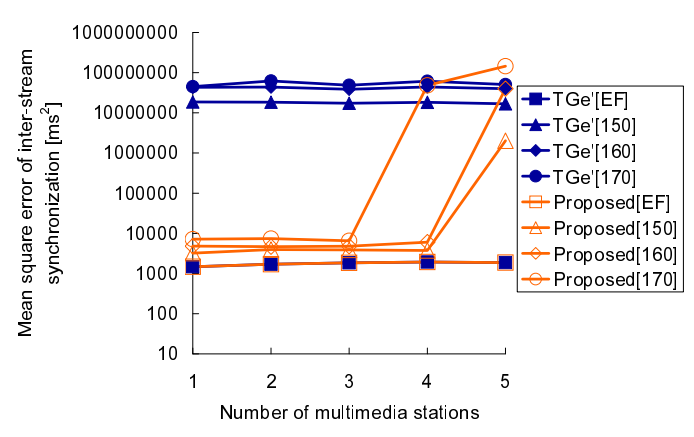

Fig. 8. Mean square error of inter-stream synchronization

TXOP duration on the basis of the number of retransmission to replenish the insufficient bandwidth. We have also discussed an admission control scheme in a noisy environment.

Our future work includes QoE estimation of the proposed scheme in multimedia transmission over IEEE 802.11e HCCA. In addition, we will examine scheduling schemes for transmission of VBR traffic in a noisy environment. We should also investigate the effects of the video coding rates and the number of slices in a video frame on the QoS.

\section{ACKNOWLEDGMENT}

This work was supported by the Grant-In-Aid for Scientific Research of Japan Society for the Promotion of Science under Grant 21360183.

\section{REFERENCES}

[1] IEEE 802.11 WG, "Specific requirements Part 11: Wireless LAN Medium Access Control (MAC) and Physical Layer (PHY) specifications: Amendment 8: Medium Access Control (MAC) Quality of Service Enhancements Amendment 7: Medium Access Control (MAC) Quality of Service (QoS) Enhancements," IEEE 802.11 Std. , 2005.

[2] A. Grilo, M. Macedo, and M. Nunes, "A scheduling algorithm for QoS support in IEEE 802.11e networks," IEEE Wireless Communications, pp.36-43, June 2003.

[3] P. Ansel, Q. Ni, and T. Tueletti, "An efficient scheduling scheme for IEEE 802.11e," in Proc. Modeling and Optimization in Mobile, Ad Hoc and Wireless Networks, WiOpt., Mar. 2004.

[4] Inanc Inan, Feyza Keceli, and Ender Ayanoglu, "An adaptive multimedia QoS scheduler for 802.11e wireless LANs" in Conf. Rec. IEEE ICC'06, June 2006.

[5] D. Xue, Y. Qin, and C. K. Siew, "A service curve based scheduling algorithm for QoS support in 802.11e wireless LANs," in Conf. Rec. IEEE GLOBECOM'06, Nov. 2006.

[6] M. M. Rashid, E. Hossain, and V. K. Bhargava, "HCCA scheduler design for guaranteed QOS in IEEE 802.11e based WLANs," in Proc. IEEE WCNC'07, Mar. 2007.

[7] A. Foronda, Y. Higuchi, C. Ohta, M. Yoshimoto, and Y. Okada, "Service interval optimization with delay bound guarantee for HCCA in IEEE 802.11e WLANs," IEICE Trans. Commun., vol. E90-8, no. 11, pp. 3158 3169, Nov. 2007.

[8] M. V. D. Schaar, Y. Andreopoulos, and Z. Hu, "Optimized scalable video streaming over IEEE 802.11a/e HCCA wireless networks under delay constraints," IEEE Trans. on Mobile Computing, vol. 5, no. 6, pp. 755-768, June 2006

[9] Z. Azri, T. Suzuki, and S Tasaka, "A multimedia priority dynamic scheduling scheme for audio-video transmission with user-level QoS guarantee by IEEE 802.11e HCCA," in Conf. Rec. IEEE PIMRC'07, Sep. 2007.

[10] IEEE 802.11 WG, "Supplement to part 11: wireless LAN medium access control (MAC) and physical layer specifications : high-speed physical layer extension in the $2.4 \mathrm{GHz}$ band," IEEE 802.11 Std., 1999.

[11] S. Tasaka and Y. Ishibashi, "Mutually compensatory property of multimedia QoS," in Conf. Rec. IEEE ICC'02, pp. 1105-1111, Apr./May 2002.

[12] W. Xiuchao, "Simulate 802.11b channel within ns2," http://www.comp.nus.edu.sg/ wuxiucha/research/reactive/publication /Simulate80211ChannelWithNS2.pdf, 2004.

[13] HFA3861B; Direct sequence spread spectrum baseband processor, Intersil, Jan 2000 